\title{
PERFIL DEL FUTURO DOCENTE: NUEVOS RETOS EN EL MARCO DEL EEES ${ }^{1}$
}

\author{
David Jiménez Hernández \\ Universidad Católica San Antonio de Murcia \\ Patricia Sancho Requena \\ Universidad de Valencia \\ Sergio Sánchez Fuentes \\ Universidad Autónoma de Madrid
}

RESUMEN: En la actualidad, los nuevos requerimientos del siglo XXI en la formación docente son evidentes. El presente trabajo tiene como principal objetivo describir el perfil de los estudiantes universitarios en temas relevantes para su futuro profesional. Entre ellos se consideran importantes los enfoques de enseñanza, la atención a la diversidad, las TIC y su relación con el cyberbullying, la inteligencia emocional y la autoestima. La investigación fue llevada a cabo en la Universidad Católica de San Antonio de Murcia (UCAM) con una muestra de 230 estudiantes de Educación. Los instrumentos utilizados para este propósito fueron el cuestionario de autoestima Rosenberg, ATI, TMMS-24 y un instrumento diseñado ad-hoc para la atención a la diversidad y el uso de las TIC y su relación con el cyberbullying. Los resultados muestran un perfil definido de estudiantes mujeres, con una alta inteligencia emocional, autoestima dentro del promedio, que no favorecen un enfoque de enseñanza específico y con medios muy elevados en la atención a la diversidad y el cyberbullying.

PALABRAS CLAVE: Enfoques docentes, atención a la diversidad, cyberbullying, inteligencia emocional, autoestima.

\section{PROFILE OF THE FUTURE TEACHER: NEW CHALLENGES IN THE FRAMEWORK OF EHEA}

ABSTRACT: Currently, the new requirements of the XXI century in teacher training are evident. This paper has as main aim to describe the profile of Education undergraduate on topics relevant to their professional future such

1 Este trabajo ha estado subvencionado por el proyecto PMAFI/16/14. 
as teaching approaches, attention to diversity, ICT and their relationship with cyberbullying, emotional intelligence and self-esteem. The research was carried out at the Catholic University of San Antonio de Murcia (UCAM) with a sample of 230 Education undergraduates. The instruments used for this purpose were the Rosenberg Self-esteem questionnaire, ATI, TMMS-24 and an instrument designed ad-hoc for attention to diversity and the use of ICT. The results show a defined profile of female students, with a high emotional intelligence, selfesteem within the average, who do not favor a specific teaching approach and with very high means in the attention to diversity and cyberbullying.

KEYWORDS: Teaching approaches, attention to diversity, cyberbullying, emotional intelligence, self-esteem.

Recibido: 27/10/2017

Aceptado: 22/12/2017

Correspondencia: David Jiménez Hernández, Universidad Católica San Antonio de Murcia, Campus de Los Jerónimos, 30107, Guadalupe, Murcia.. Email: djimenez361 @ucam.edu.

\section{INTRODUCCIÓN}

En los últimos años, la sociedad ha experimentado cambios motivados por muy diversos agentes, avances sociales y tecnológicos. En dichos cambios, la escuela ocupa un lugar preferente como plataforma de desarrollo de las nuevas generaciones. De este modo, el docente del siglo XXI ha de poder responder, con una actitud profesional, a los diferentes retos del nuevo sistema. Más concretamente, y siendo objeto de estudio en este trabajo, es necesario identificar cómo el alumnado se encuentra recibiendo formación en una enseñanza universitaria caracterizada por su inclusión en el Espacio Europeo de Educación Superior (EEES). Dicha incorporación al nuevo sistema ha supuesto grandes modificaciones con el sistema anterior. Al margen de los cambios organizativos, ahora la formación se basa en el aprendizaje activo del alumnado, tanto es así, que la medición de la enseñanza se efectúa con relación a su esfuerzo. Esto supuso un cambio radical en el enfoque de enseñanza del profesorado, exigiendo una ruptura con una cultura educativa muy arraigada a la formación docente. Trigwell y Prosser (1991) determinaron la existencia de dos enfoques, el primero, centrado en el profesorado/transmisor de conocimientos, está emparentado con la enseñanza más tradicional y conductista. Se caracteriza por tener un estilo de enseñanza controlado por el docente, construye los contenidos, es el único que toma las decisiones de cómo tienen que aprender los estudiantes e ignora sus aprendizajes previos y preparación (Bryant, 2004; Tezci, 2017). En el lado opuesto está el enfoque centrado en el alumnado, cuyo carácter es constructivista. El docente es un facilitador de la enseñanza, que involucra y motiva al alumnado en el proceso de enseñanza-aprendizaje. De acuerdo con Hynes (2017), los discentes presentan un papel activo y responsable en su proceso de aprendizaje, equiparando su importancia en el mismo con el docente. Los principales resultados de las investigaciones llevadas a cabo en esta temática, muestran como el enfoque centrado en los estudiantes se relaciona 
con el aprendizaje profundo y la calidad en el rendimiento académico (Trigwell, 2011; Baeten, Dochy y Struyven, 2013).

En la actualidad, la escuela se encuentra en la era de la educación inclusiva u orientación inclusiva (López, Martín, Montero y Echeíta, 2013). Las escuelas inclusivas se caracterizan por una filosofía de compromiso con los valores de respeto a la diversidad, a la colaboración, a la participación, a la equidad y a la solidaridad (Armstrong y Moore, 2004; Ainscow, 2006; Ainscow, Booth y Dyson, 2006). La diversidad engloba a todo el alumnado del centro y requiere que todo el profesorado diseñe actuaciones que tengan como objetivo adaptarse de manera individual a cada estudiante (Martínez, de Haro y Escarbajal, 2010).

Sin embargo, este modo de entender la atención a la diversidad sigue manteniendo premisas con un enfoque reactivo (Díez et al., 2011). Por lo tanto, uno de los retos a los que se enfrenta la formación de maestros en la actualidad, es describir enfoques que favorezcan metodologías que anticipen las posibles dificultades que se pueden presentar en el aula (Verdugo y Rodríguez, 2012). Para ello, el diseño universal representa la aplicación de un enfoque en el que, desde el primer momento, tiene en cuenta la variabilidad de personas en cualquier proceso (Spooner, Baker, Harris, Ahlgrim-Delzell y Browder, 2007). Y es que, el diseño universal aplicado a los entornos pedagógicos, puede ser un nuevo paradigma que permita hacer efectiva la implementación de la inclusión y proporcionar acceso a la educación (McGuire, Scott y Shaw, 2006; Sala, Sánchez, Giné y Díez, 2014). Trabajos relevantes en la materia (Ralabate et al., 2012; Smith, 2012; Tegmark-Chita, Gravel, Serpa, Domings y Rose, 2012; Davies, Schelly y Spooner, 2013) avalan la necesidad de formar a educadores sobre los beneficios de la aplicación del paradigma del diseño universal para el aprendizaje y sus aportaciones a los procesos de enseñanza-aprendizaje de los estudiantes. Por lo tanto, es un aspecto prioritario que la formación a futuros profesionales del ámbito educativo esté centrada en enseñar competencias que permitan atender a la diversidad del alumnado que se encuentran en la actualidad en las aulas (Díez et al., 2011).

Además, la escuela se ha considerado tradicionalmente como uno de los grandes entes sociales donde se propiciaba no solo la primera parte del proceso de socialización de las personas, sino también del desarrollo psico-evolutivo de los chicos y chicas (Pertegal, Oliva y Hernando, 2010). Por tal razón, la escuela se está viendo inmiscuida en esta transformación social provocada por las TIC como una de las grandes protagonistas (Bird y Markle, 2012). Por tanto, el comportamiento online y la participación en entornos virtuales resulta relevante en todo análisis sobre los centros educativos y sobre las dinámicas que se desarrollan en ellos, donde los jóvenes y adolescentes son los principales protagonistas (Biggs, Vernberg, Twemlow, Fonagy y Dill, 2008). En este sentido, la presencia y mediación de los dispositivos digitales y los entornos virtuales en las relaciones interpersonales de los centros educativos, podría afirmarse que ha modificado gran parte de la naturaleza de la convivencia escolar, convirtiéndose en la denominada ciberconvivencia (Ortega, Del Rey y Sánchez, 2012).

Esta mediación de dispositivos electrónicos, ha supuesto que actualmente se detecten determinadas necesidades en el contexto educativo, derivadas del uso de las redes sociales, a las que no se les está dando una respuesta (Zych, Ortega-Ruiz y Marín-López, 2016). Más concretamente, es importante dar respuesta a aquellas relacionadas la aparición de problemas y riesgos derivados del uso de las TIC, es decir, lo que se conoce como cyberbu- 
Ilying. Ese tipo de acoso puede ser ejercido y padecido en cualquier momento y lugar, lo que es conocido como 24/7, aludiendo a que la agresión o victimización es 24 horas del día los 7 días de la semana. Además, habría que añadir otros aspectos no contemplados en el bullying, como son el anonimato, que el acoso se puede producir en un ámbito tanto público como privado, mayor difusión de los daños y la reiteración que implica el empleo de las TIC (Del Rey et al., 2011). Es de vital importancia trabajar sobre el acoso, sea el tipo que sea, con futuros docentes, pues Grumm y Hein (2013) ya mostraron que los docentes cuyo alumnado percibe que se interesa por su bienestar, necesidades personales y con un enfoque consistente, contribuye a prevenir la victimización. Así mismo, Casas, Ortega-Ruiz y Del Rey (2015) también confirmaron el caso inverso, es decir, que los docentes que realizan una gestión negativa de la clase, que muestran apatía y falta de valores positivos, favorecen la victimización. Por todo ello, desde nuestra perspectiva, la comunidad educativa debe diseñar y desarrollar estrategias y actividades específicas de actuación ante determinados comportamientos de riesgo en la Red, potenciando los usos positivos y beneficiosos.

Por otro, e igual de importante, es hacer frente a las necesidades relacionadas con la Inteligencia Emocional (EI). A partir de una revisión bibliográfica, Pedrera (2017) consideró que la El está fundamentada por la dimensión personal y social, que posibilitan la gestión de sentimientos, solución de problemas y relación social (Salguero, Fernández-Berrocal, Ruiz-Aranda, Castillo y Palomera, 2011). En relación con el sistema escolar, FernándezBerrocal y Extremera apreciaron como: "los educadores son los principales líderes emocionales de sus alumnos. La capacidad del profesor para captar, comprender y regular las emociones de sus alumnos es el mejor índice del equilibrio emocional de su clase" (2002, p.6). Por tanto, se debe contar con la implicación de los docentes para aplicar la inteligencia emocional en el aula, debido a la importancia que ésta tiene para el bienestar, liderazgo, adaptación y rendimiento de los alumnos (Lam y Kirby, 2002; Petrides, Frederikson y Furnham, 2004; Extremera y Fernández-Berrocal, 2006; Downey, Mountstephen, Lloyd, Hansen y Stough, 2008; Siu, 2009; Villanueva y Sánchez, 2007, citado en Fernández, Bermejo, Sainz, Llor, Hernández y Soto, 2011). Además, Extremera y Fernández-Berrocal (2004) afirman que la inteligencia emocional es un conjunto de habilidades que el docente debería aprender por dos razones: (1) porque las aulas son el modelo de aprendizaje socio-emocional adulto de mayor impacto para los discentes y; (2) porque unos adecuados niveles de inteligencia emocional ayudan a afrontar con mayor éxito los contratiempos cotidianos y el estrés laboral al que se enfrenta el profesorado en el contexto educativo.

Finalmente, como una característica también importante dentro del perfil docente de los futuros maestros, encontramos la autoestima. Dicha característica es considerada como una protectora de la salud mental (Góngora y Casullo, 2009; Ros, Filella, Ribes y Pérez, 2017) y de vital importancia en el ámbito educativo. Además, ésta se ha considerado relevante para predecir (a) el éxito académico de los estudiantes (Mestre, García, Frías y Llorca, 1992); (b) las expectativas de los profesores con respecto a la realización de las tareas escolares (Burns, 1982); (c) la correlación entre la autoestima académica del estudiante, rendimiento escolar y ajuste psicosocial (Rodríguez, 1982); o (d) el papel protector frente al estrés en estudiantes (Cabanach, Souto, Freire y Ferradás, 2014). Incluso, y siendo mucho más actual, la autoestima ha resultado ser una variable predictora de bullying, al mostrar sus víctimas un nivel significativamente inferior de autoestima (Suresh y Tipandjan, 2012; Garaigordobil, Martínez-Valderrey y Aliri, 2013). 
Ante este panorama, por tanto, los futuros docentes tendrán un papel fundamental a la hora de desarrollar las competencias socio-emocionales de sus alumnos, enseñándoles a conocer sus sentimientos, la forma de manejarlos, así como fomentar la capacidad de enfrentarse a las dificultades positivamente. Estas consideraciones nos remiten a la necesidad de integrar dichas competencias en el plan de estudios de Magisterio (Bueno, Teruel y Valero, 2005).

Por todo ello, este trabajo tiene como objetivo principal identificar el perfil docente de un grupo de estudiantes de magisterio sobre las cuestiones mencionadas con anterioridad en este trabajo.

\section{Método}

\section{Participantes y procedimiento}

Con el fin de recopilar información que permitiera construir un perfil tipo del estudiante de Magisterio, y detectar necesidades formativas, se optó por un diseño transversal. La obtención de los datos se realizó de dos maneras, mediante la realización de los cuestionarios on-line a través del servicio proporcionado por SurveyMonkey o bien en papel.

La muestra total del estudio estuvo formada por 230 alumnos universitarios, un $60 \%$ de estudiantes de Grado de Educación Infantil y un $40 \%$ de estudiantes de Grado de Educación Primaria. La distribución por cursos se puede observar en la Figura 1. Con una edad media de 22.38 años (DT $=4.06$ ), el $72.2 \%$ eran mujeres, frente a un $24.8 \%$ de hombres.

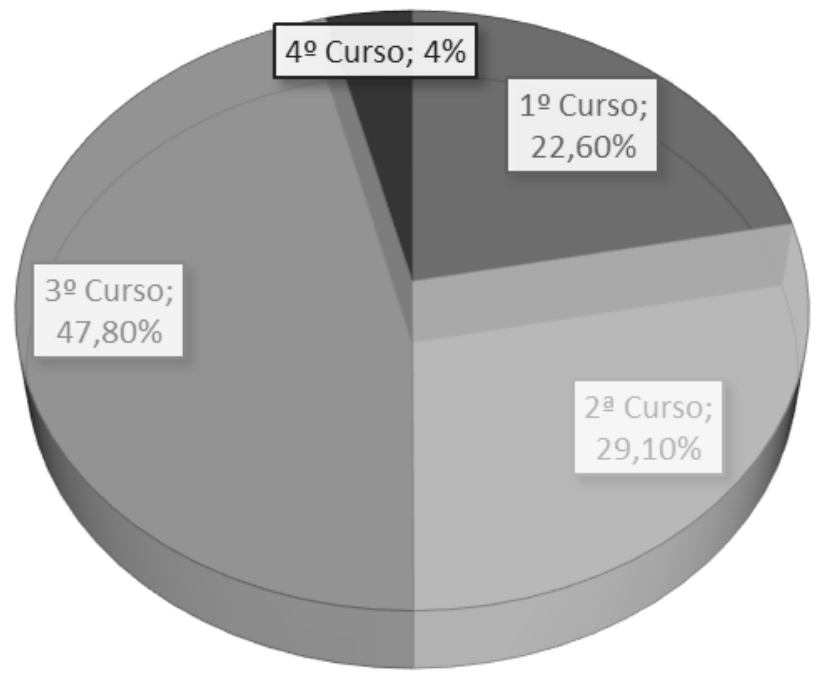

Figura 1. Distribución por curso de la muestra 


\section{Instrumentos}

El instrumento de medida para realizar la investigación constó de dos partes que se desarrollaron de forma simultánea, siguiendo un procedimiento de autoinforme, siempre con la supervisión y el apoyo de algún miembro del equipo, para la resolución de dudas que los participantes puedan tener al respecto.

En primer lugar, además de recoger información relativa a variables sociodemográficas, se incluyó un cuestionario diseñado ad-hoc para recabar información relativa al conocimiento de los futuros docentes en atención a la diversidad y TIC y el ciberacoso, así como las actuaciones que un maestro puede, y debe, llevar a cabo a este respecto.

En segundo lugar, se incluyeron una serie de cuestionarios estandarizados una serie de constructos de interés para la investigación:

- Inteligencia Emocional, medida mediante el cuestionario TMMS-24 (Fernández-Berrocal, Extremera y Ramos, 2004; versión original de Salovey et al., 1995), con un total de 24 ítems que forman las dimensiones de percepción $(\mathrm{a}=.858)$, comprensión $(\mathrm{a}=.849)$ y regulación $(\mathrm{a}=.741)$.

- Autoestima, utilizando el cuestionario de Autoestima de Rosenberg (Rosenberg, 1965), que cuenta con un total de 10 ítems que forman un solo factor $(\mathrm{a}=.668)$.

- Enfoques docentes, medidos a través del ATI, que diferencia entre dos dimensiones, enfoque centrado en el alumno $(a=.702)$ y enfoque centrado en el alumno $(\mathrm{a}=.729)$, con un total de 13 ítems.

- Conocimiento del profesorado en Cyberbullying (Li, 2008), medido con el cuestionario creado para tal efecto, con 22 ítems que forman un solo factor $(\mathrm{a}=.872)$.

\section{Análisis}

Además de estadísticos descriptivos (medias y desviaciones típicas), se llevaron a cabo diferentes análisis teniendo en cuenta la naturaleza de las variables implicadas en el estudio: Pruebas t, MANOVAs y correlaciones de Pearson. Estas últimas se interpretaron siguiendo a Cohen (1988), dichas correlaciones se interpretaron como pequeñas $(r=.10)$, moderadas $(r=.30)$ y elevadas $(r \geq .50)$.

Se incluyó el cálculo de tamaños del efecto ( $\eta^{2}$ parciales) basándose en Cohen (1992) para determinar el efecto bajo $\left(\eta^{2}=.02\right)$, moderado $\left(\eta^{2}=.13\right)$ y elevado $\left(\eta^{2}=.26\right)$.

\section{Resultados}

En la Tabla 1 se pueden observar los resultados descriptivos relativos a constructos psicológicos o relacionados, tales como atención a la diversidad, cyberbullying, inteligencia emocional y autoestima. En todos los casos, las variables de estudio cuentan con medias superiores al punto medio de la escala en todos los casos. 
Tabla 1. Estadísticos descriptivos de constructos psicológicos y relacionados

\begin{tabular}{llcccc}
\hline & & Mínimo & Máximo & Media & Desviación Típica \\
\hline \multirow{2}{*}{$\begin{array}{l}\text { Inteligencia } \\
\text { Emocional }\end{array}$} & Autoestima & 1,90 & 6.00 & 3.19 & .50 \\
\cline { 2 - 6 } & Percepción & 2.30 & 5.00 & 3.72 & .65 \\
\cline { 2 - 6 } & Comprensión & 1.50 & 5.00 & 3.78 & .64 \\
\hline \multirow{2}{*}{$\begin{array}{l}\text { Atención a la lación } \\
\text { diversidad }\end{array}$} & $\begin{array}{l}\text { Múltiples formas de re- } \\
\text { presentación }\end{array}$ & 1.20 & 4.00 & 3.56 & .36 \\
\cline { 2 - 6 } & $\begin{array}{l}\text { Múltiples formas de ex- } \\
\text { presión y acción }\end{array}$ & 1.75 & 4.00 & 3.37 & .37 \\
\cline { 2 - 6 } & $\begin{array}{l}\text { Múltiples formas de parti- } \\
\text { cipación }\end{array}$ & 1.33 & 4.00 & 3.58 & .39 \\
\hline Ciberbullying & 1.91 & 5.00 & 4.22 & .48 \\
\hline
\end{tabular}

Los resultados descriptivos de constructos relativos a competencias docentes aparecen en la Tabla 2. En primer lugar, las medias de los enfoques docentes son muy similares en ambas dimensiones, con una media ligeramente superior en el caso del enfoque centrado en el alumno.

En segundo lugar, con respecto a las competencias tecnológicas, los resultados muestran medias elevadas en los ítems relativos al dominio de este tipo de competencias. En todos los casos, las medias más bajas se dan en los ítems relacionados con experimentar problemas con la tecnología (por ejemplo, ítem 2 e ítem 10), a excepción del ítem que hace referencia a usar las tecnologías para jugar, que cuenta con la media más baja.

Tabla 2. Estadísticos descriptivos relativos a constructos relacionados con competencias docentes

\begin{tabular}{|c|c|c|c|c|}
\hline & Mínimo & Máximo & Media & $\begin{array}{l}\text { Desviación } \\
\text { típica }\end{array}$ \\
\hline \multicolumn{5}{|l|}{ Enfoque docente } \\
\hline Centrado en el Alumno & 1.83 & 4.00 & 3.364 & .411 \\
\hline Centrado en el profesor & 1.50 & 4.00 & 3.047 & .541 \\
\hline \multicolumn{5}{|l|}{ Competencias Tecnológicas } \\
\hline 1. Me resulta fácil utilizar el correo & 1.00 & 5.00 & 4.677 & .651 \\
\hline $\begin{array}{l}\text { 2. El uso del software de mensajería, } \\
\text { como GMAIL, siempre me causa algunos } \\
\text { problemas. }\end{array}$ & 1.00 & 5.00 & 2.201 & 1.305 \\
\hline $\begin{array}{l}\text { 3. Tendría pocos problemas para instalar } \\
\text { una web cam. }\end{array}$ & 1.00 & 5.00 & 3.469 & 1.343 \\
\hline $\begin{array}{l}\text { 4. Para comunicarme con la gente prefiero } \\
\text { Ilamar, en lugar de internet o WhatsApp. }\end{array}$ & 1.00 & 5.00 & 3.231 & 1.332 \\
\hline $\begin{array}{l}\text { 5. Los grupos de WhatsApp son una bue- } \\
\text { na manera de llegar a la gente. }\end{array}$ & 1.00 & 5.00 & 3.528 & 1.208 \\
\hline
\end{tabular}




\begin{tabular}{lcccc}
\hline & Mínimo & Máximo & Media & $\begin{array}{c}\text { Desviación } \\
\text { típica }\end{array}$ \\
\hline $\begin{array}{l}\text { 6. Tengo poca idea de para qué sirve el } \\
\text { software peer to peer. }\end{array}$ & 1.00 & 5.00 & 3.388 & 1.539 \\
\hline $\begin{array}{l}\text { 7. Intercambio regularmente con mis ami- } \\
\text { gos archivos de música y/o videos. }\end{array}$ & 1.00 & 5.00 & 3.733 & 1.186 \\
\hline $\begin{array}{l}\text { 8. Usar internet hace que sea mucho más } \\
\text { fácil mantenerse en contacto con la gente. }\end{array}$ & 1.00 & 5.00 & 4.196 & .950 \\
\hline $\begin{array}{l}\text { 9. Uso regularmente internet para jugar. } \\
\text { 10. No estoy seguro de cómo comunicarme } \\
\text { con personas que utilizan las redes sociales. }\end{array}$ & 1.00 & 5.00 & 2.535 & 1.311 \\
\hline
\end{tabular}

Cuando se hace un estudio inferencial de las variables en base a la edad (Tabla 3), se observa que ésta no es determinante, a excepción del caso de la dimensión de Comprensión de la Inteligencia Emocional, en la que se obtiene una correlación positiva.

Tabla 3. Correlaciones de las variables de estudio con edad

\begin{tabular}{|c|c|c|c|c|c|c|c|c|c|c|}
\hline & \multicolumn{2}{|c|}{$\begin{array}{l}\text { Enfoque } \\
\text { Docente }\end{array}$} & \multicolumn{3}{|c|}{$\begin{array}{c}\text { Atención a la } \\
\text { Diversidad }\end{array}$} & \multirow[t]{2}{*}{ Autoestima } & \multicolumn{3}{|c|}{ Inteligencia Emocional } & \multirow{2}{*}{ Ciberbullying } \\
\hline & $\mathrm{ECA}$ & $\mathrm{ECP}$ & ADF1 & ADF2 & ADF3 & & Percepción & Comprensión & Regulación & \\
\hline Edad & .082 & -.018 & -.011 & .053 & .096 & .037 & -.085 & $.185^{* *}$ & .097 & -.065 \\
\hline
\end{tabular}

Con respecto a los análisis inferenciales (Tabla 4), no se hallaron diferencias estadísticamente significativas en función del género en los enfoques, atención a la diversidad ni autoestima $\left(t_{227}=-.730, p=.466\right)$. No obstante, el género sí muestra su determinación en el caso de la inteligencia emocional, concretamente de la percepción, con una media de 3.75 en mujeres y de 3.47 en hombres. Del mismo modo, influye en el caso del cyberbullying $\left(t_{227}=3.164, p=.002\right)$, con una media de 4.28 en mujeres y 4.03 en hombres.

Tabla 4. MANOVAs en función del género en constructos psicológicos

\begin{tabular}{llcccc}
\hline & & $\boldsymbol{F}$ & $\mathbf{G l}$ & $\boldsymbol{p}$ & $\boldsymbol{\eta}^{\mathbf{2}}$ \\
\hline Enfoques docentes & 1.620 & 2,226 & .200 & - \\
\hline Atención a la Diversidad & & 2.053 & 3,226 & .107 & - \\
\hline Inteligencia Emocional & & 4.507 & 3,225 & .004 & .057 \\
\hline \multirow{3}{*}{ ANOVAs de continuación } & Percepción & 8.172 & 1,227 & .005 & .030 \\
\cline { 2 - 6 } & Comprensión & .593 & 1,227 & .442 & - \\
\cline { 2 - 6 } & Regulación & 1.256 & 1,227 & .264 & - \\
\hline
\end{tabular}

Siguiendo con los contrastes en función del género, también se hallaron diferencias estadísticamente significativas en competencias $\left(F_{9,221}=2.172, p=.025, \mathrm{~h}^{2}=\right.$ 
.085). Aunque únicamente se evidenciaron diferencias estadísticamente significativas en los ítems 3 y 6 (Tabla 5). En el ítem 3, relativo a las competencias para instalar una webcam, las mujeres obtienen una media de 3.335 (DT = .102) y los hombres una media de $1.907(\mathrm{DT}=.180)$. En el ítem 6, relativo al conocimiento del software peer to peer, las mujeres obtienen una media de $3.53(\mathrm{DT}=118)$ y los hombres de 3.05 $(\mathrm{DT}=207)$.

Tabla 5. ANOVAs de continuación de competencias tecnológicas en función del género

\begin{tabular}{|c|c|c|c|c|}
\hline & $F$ & GI & $p$ & $\eta^{2}$ \\
\hline 1. Me resulta fácil utilizar el correo & .456 & 1,219 & .500 & - \\
\hline $\begin{array}{l}\text { 2. El uso del software de mensajería, como GMAIL, siempre } \\
\text { me causa algunos problemas }\end{array}$ & 3.192 & 1,219 & .075 & - \\
\hline 3. Tendría pocos problemas para instalar una webcam & 6.674 & 1,219 & .010 & .030 \\
\hline $\begin{array}{l}\text { 4. Para comunicarme con la gente prefiero llamar, en lugar } \\
\text { de internet o WhatsApp }\end{array}$ & .634 & 1,219 & .427 & - \\
\hline $\begin{array}{l}\text { 5. Los grupos de WhatsApp son una buena manera de llegar } \\
\text { a la gente }\end{array}$ & 2.153 & 1,219 & .144 & - \\
\hline 6. Tengo poca idea de para qué sirve el software peer to peer & 4.006 & 1,219 & .047 & .018 \\
\hline $\begin{array}{l}\text { 7. Intercambio regularmente con mis amigos archivos de } \\
\text { música y/o videos }\end{array}$ & .296 & 1,219 & .587 & - \\
\hline $\begin{array}{l}\text { 8. Usar internet hace que sea mucho más fácil mantenerse } \\
\text { en contacto con la gente }\end{array}$ & .436 & 1,219 & .510 & - \\
\hline 9. Uso regularmente internet para jugar & 3.501 & 1,219 & .063 & - \\
\hline $\begin{array}{l}\text { 10. No estoy seguro de cómo comunicarme con personas } \\
\text { que utilizan las redes sociales }\end{array}$ & .456 & 1,219 & .500 & - \\
\hline
\end{tabular}

\section{Discusión}

En base a estos resultados obtenidos, se puede dibujar un perfil del estudiante de magisterio. Se trataría de una mujer, de entorno a los 22 años, que pese a enmarcar su educación y futuro profesional dentro del marco del EEES, no parece decantarse por un enfoque de enseñanza en concreto, aunque sí destaca en cierto modo el enfoque centrado en el alumno (aunque con una diferenciación mínima). Esto puede ser debido, a que como resalta Postareff (2007), el profesorado puede emplear indistintamente los dos enfoques, principalmente, por la complejidad de la educación superior. Otros como Kain (2003) consideran importante no emplear un único enfoque, para que en el proceso de aprendizaje exista una mayor diversidad y esté dirigido en ambos agentes.

Sin embargo, cuando se trata de atender a la diversidad y cyberbullying, los alumnos de la educación se encuentran especialmente comprometidos, con unas medias extraordinariamente elevadas. Esto se relacionaría directamente con lo expuesto por algunas tesis, basadas en que la formación recibida sobre atención a la diversidad mejora el conocimiento y la autopercepción de su futuro trabajo como docentes y las necesidades educativas especiales (Sánchez, Díez y Martín, 2015). Especialmente, 
aquellas medidas de educación inclusiva basadas en la premisa del diseño universal para el aprendizaje.

Una primera conclusión que se puede extraer del análisis de los resultados, es que los programas docentes sí que tienen una repercusión directa sobre la formación y sobre los conocimientos que los estudiantes adquieren durante su etapa universitaria. Como ejemplo de que esto no se está haciendo de la mejor manera, hay que destaca un artículo publicado recientemente donde se describe que menos de la mitad de las universidades españolas están enseñando contenidos relacionados con la accesibilidad y el diseño para todos (Sánchez, Díez y Almaraz, 2016). Sin embargo, otras experiencias que se están Ilevando a cabo en nuestro país, tienen un carácter divulgador mucho mayor que servirá para obtener herramientas que permitan el acceso al conocimiento sobre educación inclusiva a los futuros docentes. Con esto nos referimos a la publicación del libro sobre cómo enseñar diseño para todos en las carreras de Magisterio (CRUE, 2017).

Además, este alumnado muestra una elevada inteligencia emocional y una autoestima dentro de la media. Dicha inteligencia emocional, se vincula con unos buenos niveles de ajuste psicológico (Extremera y Fernández-Berrocal, 2003). Siguiendo con lo anterior, a través de la cita ya expuesta de Fernández-Berrocal y Extremera (2002), dada las puntuaciones obtenidas en los altos niveles de inteligencia emocional, los futuros docentes van a poder obtener un buen índice del equilibrio emocional de sus clases. En este estudio no se puede mostrar, como en el de Salovey, Stroud Woolery y Epel (2002), que una elevada inteligencia emocional se relacione con una alta autoestima. Puesto que las puntuaciones en este último se encontraban en la media.

De esta manera, trabajos como la reciente publicación de Cejudo y López-Delgado (2017) donde se afirman que existe una necesidad de conocimiento sobre su propia inteligencia emocional como maestros para lograr un buen desempeño laboral, se podrían correlacionar con los resultados expuestos en este trabajo y extraer la conclusión de la importancia de la formación sobre dichos aspectos en los primeros años de formación académica en educación, en asignaturas como psicología del desarrollo o psicología educativa.

En general, los resultados obtenidos muestran que el perfil del futuro docente obtiene muy buenos resultados en cuestiones prioritarias para la enseñanza de calidad como son, la atención a la diversidad, alfabetización digital, la inteligencia emocional que éstos presentan, y su capacidad de adaptación a los nuevos enfoques de enseñanza que el sistema, y su población, requieren. Por tanto, el modelo planteado por la universidad española podría servir de ejemplo para otras universidades que, evaluadas en futuras investigaciones en constructos semejantes, muestren resultados deficitarios en alguno de los aspectos que forman los retos de la nueva Educación.

Por otro lado, los autores destacan como limitaciones generales halladas en el estudio, la imposibilidad de acceder a otras universidades de la región para haber podido realizar un estudio más amplio y representativo de la población, así como el sesgo producido por la existencia de un elevado número de mujeres en el estudio. Si bien esta circunstancia suele darse en el número de profesorado en la Educación Pri- 
maria e Infantil, también es cierto, que dicha tendencia se está invirtiendo en los últimos tiempos, en especial, en especialidades como es la de Educación Física o Inglés.

A modo de conclusión general, los autores destacan, que como se viene publicando en la literatura científica actual (Montero, Martínez-Piñeiro y Colén, 2016; Veloquio, 2016) que, además, de lo sugerido en este trabajo, los futuros docentes tendrán que trabajar siguiendo la premisa de aprendizaje a lo largo de toda la vida, ya que la formación única recibida, no garantizará un buen desempeño laboral futuro si no se producen actualizaciones e incorporaciones de nuevos conocimientos a su práctica en el aula.

\section{REFERENCIAS BIBLIOGRÁFICAS}

Ainscow, M. (2006). From special education to effective schools for all: a review of progress so far. En L. Floiran (Ed.), The Sage Handbook os Special Education (pp. 146-159). London: Sage.

Ainscow, M., Booth, T. y Dyson, A. (2006). Improving Schools, Developing Inclusion. London: Routledge.

Armstrong, F. y Moore, M. (2004). Action Research for Inclusive Education: Changing Places, Changing Practices, Changing Minds. London: RoutledgeFalmer.

Baeten, M., Dochy, F. y Struyven, K. (2013). Enhancing students' approaches to learning: the added value of gradually implementing case-based learning. European journal of psychology of education, 28(2), 315-336.

Biggs, B. K., Vernberg, E. M., Twemlow, S. W., Fonagy, P. y Dill, E. J. (2008). Teacher adherence and its relation to teacher attitudes and student outcomes in an elementary school-based violence prevention program. School Psychology Review, 37(4), 533.

Bird, J. M. y Markle, R. S. (2012). Subjective Well-Being in School Environments: Promoting Positive Youth Development Through Evidence-Based Assessment and Intervention. American Journal of Orthopsychiatry, 82(1), 61-66. http://doi. org/10.1111/j.1939-0025.2011.01127.x.

Bryant, P. A. (2004). Teaching styles of interactive television instructors: A descriptive study. American Journal of Distance Education, 18(1), 39-50. http://doi. org/10.1207/s15389286ajde1801_4.

Bueno C., Teruel, P. y Valero, A. (2005). La inteligencia emocional en alumnos de Magisterio: la percepción y comprensión de los sentimientos y las emociones. Revista Interuniversitaria de Formación del Profesorado, 19(3), 169-194.

Burns, R. (1982). Self-Concept Development and Education. Holt: Rinehart and Winston.

Cabanach, G., Souto, A., Freire, C. y Ferradás, M. M. (2014). Relaciones entre autoestima y estresores percibidos en estudiantes universitarios. European Journal of Education and Psychology, 7(1), 41-55. Recuperado de: http://www.redalyc.org/ articulo.oa? id=129330657004. 
Casas, J. A., Ortega-Ruiz, R. y Del Rey, R. (2015). Bullying: The impact of teacher management and trait emotional intelligence. British journal of educational psychology, 85(3), 407-423.

Cejudo, J. y López-Delgado, M. L. (2017). Importancia de la inteligencia emocional en la práctica docente: un estudio con maestros. Psicología Educativa, 23, 29-36. http://doi.org/10.1016/j.pse.2016.11.001.

Conferencia Rectores de las Universidades Españolas (2017). Formación curricular en diseño para todas las personas. Educación. Madrid: CRUE.

Davies, P., Schelly, C. y Spooner, C. (2013). Measuring the Effectiveness of Universal Design for Learning Intervention in Postsecondary Education. Journal of Postsecondary Education and Disability, 26(3), 195-220.

Del Rey, R., Flores, J., Garmendia, M., Martínez, G., Ortega, R. y Tejerina, O. (2011). Protocolo de actuación escolar ante el cyberbullying. Bilbao: Grupo EMICI.

Díez, E., Alonso, A., Verdugo, M.A., Campo, I., Sancho, I., Sánchez, S., ... Moral, E. (2011). Espacio Europeo de Educación Superior: estándares e indicadores de buenas prácticas para la atención a estudiantes universitarios con discapacidad. Salamanca: Instituto Universitario de Integración en la Comunidad. Universidad de Salamanca.

Extremera, N. y Fernández-Berrocal, P. (2004). La importancia de desarrollar la inteligencia emocional en el profesorado. Revista Iberoamericana de Educación, 33,1-10.

Extremera, N. y Fernández-Berrocal, P. (2003). La inteligencia emocional en el contexto educativo: hallazgos científicos de sus efectos en el aula. Revista de Educación, 332, 97-116.

Fernández, M. C., Bermejo, R., Sainz, M., Llor, L., Hernández, D. y Soto, G. (2011). Percepción socioemocional de los profesores en adolescentes con altas habilidades versus habilidades medias. Revista Electrónica Interuniversitaria de Formación del Profesorado, 14(3), 55-64.

Fernández-Berrocal, P. y Extremera, N. (2002). La inteligencia emocional como una habilidad esencial en la escuela. Revista Iberoamericana de Educación, 29, 1-6.

Garaigordobil, M., Martínez-Valderrey, V. y Aliri, J. (2013). Autoestima, empatía y conducta agresiva en adolescentes víctimas de bullying presencial. European Journal of investigation in health, psychology and education, 1, 29-40. Recuperado de: https://www.researchgate.net/publication/258316930.

Góngora, V. C. y Casullo, M. M. (2009). Validación de la escala de autoestima de Rosenberg en población general y en población clínica de la ciudad de Buenos Aires. Revista Iberoamericana de Diagnóstico y Evaluación Psicológica, 1, 179-194.

Grumm, M. y Hein, S. (2013). Correlates of teachers' ways of handling bullying. School PsychologyInternational,34,299-312.http://doi.org/10.1177/0143034312461467. 
Hynes, M. (2017). Students-as-producers: Developing valuable student-centered research and learning opportunities. International Journal of Research Studies in Education, 7(4). http://doi.org/10.5861/ijrse.2017.1858.

Kain, D. J. (2003). Teacher-Centered versus Student-Centered: Balancing Constraint and Theory in the Composition Classroom. Pedagogy, 3(1), 104-108. http://doi. org/10.1215/15314200-3-1-104.

Li, Q. (2008). Cyberbullying in schools: An examination of preservice teachers' perception. Canadian Journal of Learning and Technology, 34(2). Recuperado de https://www.learntechlib.org/p/42831/.

López, M., Martín, E., Montero, I. y Echeíta, G. (2013). Concepciones psicopedagógicas sobre los procesos de inclusión educativa: variables que las modulan y perfiles que las agrupan. Infancia y Aprendizaje: Journal for the Study of Education and Development, 36(4), 455-472. http://doi.org/10.1174/021037013808200285.

Martínez, R., De Haro, R. y Escarbajal, A. (2010). Una aproximación a la educación inclusiva en España. Resvista Educación Inclusiva, 3(1), 149-164.

McGuire, J., Scott, S. y Shaw, S. (2006). Universal Design and Its Aplications in Educational Environments. Remedial and Special Education, 27(3), 166-175.

Mestre, M. V., García, R., Frías, D. y Llorca, V. (1992). Autoestima, depresión y variables escolares: un estudio longitudinal infancia-adolescencia. Revista de Psicología de la Educación, 4, 51-65.

Montero, L., Martínez Piñeiro, E. y Colén, M., (2017). Los estudios de Grado en la formación inicial de Maestros en Educación Primaria. Miradas de formadores y futuros maestros. Profesorado. Revista de Currículum y Formación de Profesorado, 21(1), 1-16. Recuperado de https://recyt.fecyt.es/index.php/profesorado/article/ view/58041.

Ortega, R., Del Rey, R. y Sánchez, V. (2012). Nuevas dimensiones de la convivencia escolar y juvenil. Ciberconducta y relaciones en la Red: Ciberconvivencia. Madrid: Ministerio de Cultura.

Pedrera, M. I. (2017). Competencias emocionales y rendimiento académico en Centros de Educación Primaria de la Red Extremeña de Escuelas de Inteligencia Emocional (Tesis doctoral, Universidad de Extremadura, Cáceres). Recuperada de http://hdl.handle.net/10662/6034.

Pertegal, M. Á., Oliva, A. y Hernando, A. (2010). Los programas escolares como promotores del desarrollo positivo adolescente. Cultura y Educación, 22(1), 53-66. http://doi.org/10.1174/113564010790935169.

Postareff, L. (2007). Teaching in Higher Education: From Content-focused to Learningfocused approaches to teaching (Tesis doctoral, University of Helsinki). Recuperada de: http://helda.helsinki.fi/handle/10138/19882.

Ralabate, P., Dodd, E., Vue, G., Karger, J., Smith, F. y Carlisle, A. (2012). Universal Design for Learning (UDL): Initiatives on the Move. Understanding the impact of 
the race to the top and ARRA Funding on the Promotion of Universal Design for Learning (p. 64). Wakefield, MA: Center for Applied Special Technology.

Rodríguez, S. (1982). Factores de rendimiento escolar. Barcelona: OikosTau.

Ros, A., Filella, G., Ribes, R. y Pérez, N. (2017). Análisis de la relación entre competencias emocionales, autoestima, clima de aula, rendimiento académico y nivel de bienestar en educación primaria. Revista Española de Orientación y Psicopedagogía, 28(1). Recuperado de http://www.redalyc.org/articulo.oa?id=338252055002.

Sala, I., Sánchez, S., Giné, C. y Díez, E. (2014). Análisis de los distintos enfoques del paradigma del diseño universal aplicado a la educación. Revista Iberoamericana de Educación Inclusiva, 8(1), 143-152.

Salguero, J. M., Fernández-Berrocal, P., Ruiz-Aranda, D., Castillo, R. y Palomera, R. (2011). Inteligencia emocional y ajuste psicosocial en la adolescencia: El papel de la percepción emocional. European Journal of Education and Psychology, 4(2), 143-152.

Salovey, P., Stroud, L.R., Woolery, A. y Epel, E.S. (2002). Perceived emotional intelligence, stress reactivity, and symptom reports: Further explorations using the Trait Meta-Mood Scale. Psychology and Health, 17, 611-627. http://doi. org/10.1080/08870440290025812.

Sánchez, S., Díez, E. y Almaraz, R.A. (2016). La implementación de contenidos sobre diseño universal en ingeniería informática y arquitectura en España. Psychology, Society and Education, 8(1), 53-64.

Sánchez, S., Díez, E. y Martín, R. (2015). El diseño universal como medio para atender a la diversidad en la educación. Una revisión de casos de éxito en la universidad. Contextos Educativos. Revista de Educación, 19, 121-131. http://doi. org/10.18172/con.2752.

Smith, F. (2012). Analyzing a college course that adheres to the Universal Design for Learning (UDL) framework. Journal of the Scholarship of Teaching and Learning, 12(3), 31-61.

Spooner, F., Baker, J., Harris, A., Ahlgrim-Delzell, L. y Browder, D. (2007). Effects of Training in Universal Design for Learning on Lesson Plan Development. Remedial and Special Education, 28(2), 108-116. http://doi.org/10.1177/07419325070280 020101.

Suresh, S. y Tipandjan, A. (2012). School bullying victimization and college adjustment. Journal of the Indian Academy of Applied Psychology, 38(1), 68-73.

Tegmarck-Chita, M., Gravel, J.W., Serpa, M., Domings, Y. y Rose, D. (2012). Using the Universal Design for Learning framework to support culturally diverse learners. Journal of Education, 192(1), 17-22.

Tezci, E. (2017). Adaptation of ATI-R Scale to Turkish Samples: Validity and Reliability Analyses. International Education Studies, 10(1), 67-81. 
Trigwell, K. (2011). Scholarship of teaching and teachers' understanding of subject matter. International Journal for the Scholarship of Teaching and Learning, 5(1). doi.org/10.20429/ijsotl.2011.050101.

Trigwell, K. y Prosser, M. (1991). Relating approaches to study and the quality of learning outcomes at the course level. British Journal of Educational Psychology, 61, 265-275. http://doi.org/10.1111/j.2044-8279.1991.tb00984.x.

Veloquio, G. (2016). La formación permanente de los docentes, ante el desafío de atender a la diversidad educativa. Revista de educación inclusiva. 9(2), 144-154.

Verdugo, M.A. y Rodríguez, A. (2012). La inclusión educativa en España desde la perspectiva de alumnos con discapacidad intelectual, de familias y de profesionales. Revista de Educación, (358), 450-470. http://doi.org/10.4438/1988-592XRE-2011-358-086.

Zych, I., Ortega-Ruiz, R. y Marín-López, I. (2016). Cyberbullying: a systematic review of research, its prevalence and assessment issues in Spanish studies. Psicología Educativaa Educativa, 22(1), 5-18. https://doi.org/10.1016/j.pse.2016.03.002. 